Artículo

\title{
Aptitud combinatoria general y específica de híbridos varietales de maíz amarillo de baja endogamia
}

\author{
Consuelo López-López ${ }^{1}$ \\ Margarita Tadeo-Robledo ${ }^{2}$ \\ J. Jesús García-Zavala ${ }^{1}$ \\ Alejandro Espinosa-Calderón ${ }^{3}$ \\ J. Apolinar Mejía-Contreras ${ }^{1 \S}$
}

${ }^{1}$ Programa de Genética-Campus Montecillo-Colegio de Postgraduados. Carretera México-Texcoco km 36.5, Montecillo, Texcoco, Estado de México. CP. 56230. (lopez8con@gmail.com; zavala@colpos.mx). ${ }^{2}$ Facultad de Estudios Superiores Cuautitlán-UNAM. Carretera Cuautitlán-Teoloyucan km 2.5, Cuautitlán Izcalli, Estado de México. CP. 54714. (tadeorobledo@yahoo.com). ${ }^{3}$ Campo Experimental Valle de MéxicoINIFAP. Carretera Los Reyes-Texcoco km 13.5, Coatlinchán, Texcoco, Estado de México, México. CP. 56250. (espinoale@yahoo.com.mx).

Autor para correspondencia: mapolina@ colpos.mx.

\section{Resumen}

La producción de maíz (Zea mays L.) amarillo en México es deficitaria y su demanda aumenta cada año. En este trabajo se estimaron los efectos de aptitud combinatoria general, específica, efectos maternos y recíprocos para las variables de rendimiento y sus componentes de cinco variedades de maíz amarillo de bajo nivel de endogamia y sus cruzas dialélicas. Todos los genotipos se evaluaron en un diseño experimental de bloques completos al azar con tres repeticiones por ambiente, durante el ciclo primavera-verano de 2017 a 2019. El análisis genético se realizó usando el método I de Griffing. Los resultados indicaron que la variedad IA324 por su alto rendimiento tendría una alta contribución en la expresión del rendimiento de su progenie y podría incluirse en un programa de mejoramiento genético de maíz. Las cruzas directa y recíproca con mayor aptitud combinatoria específica (ACE) para rendimiento fueron HVAA-10 x IA324 e IA324 x HVAA-9, respectivamente. Se comprobó que el empleo de progenitores de aptitud combinatoria general (ACG) contrastante permitió la expresión favorable de sus progenies. Las variedades de grano amarillo de este trabajo con altos efectos de ACG pueden emplearse para desarrollar variedades sintéticas o seguir avanzándolas más ciclos de selección, teniendo en cuenta que las líneas con alta ACG detectadas en pruebas tempranas conservan sus valores aditivos en generaciones avanzadas, mientras que las cruzas con alta ACE se pueden usar para hibridación.

Palabras clave: Zea mays L., aptitud combinatoria general, aptitud combinatoria específica, híbridos varietales.

Recibido: marzo de 2021

Aceptado: mayo de 2021 


\section{Introducción}

En la región comprendida por los Valles Altos del centro de México, constituidos por la Ciudad de México, Hidalgo, Puebla, Tlaxcala y el Estado de México, durante los ciclos primavera verano de los años 2017 a 2019, se sembraron 1.4 millones de hectáreas con maíz (Zea mays L.). De esos estados, solo Hidalgo, el Estado de México y Puebla siembran maíz de grano amarillo bajo condiciones de temporal, en una superficie aproximada de 2786 hectáreas, con un rendimiento promedio de $2.9 \mathrm{t} \mathrm{ha}^{-1}$ (SIAP, 2020).

El rendimiento nacional promedio de maíz amarillo bajo la condición de temporal en 2019 fue de $3.9 \mathrm{t} \mathrm{ha}^{-1}$ y bajo riego de $9.9 \mathrm{t} \mathrm{ha}^{-1}$ (SIAP, 2020). En contraste, el rendimiento de maíz de grano amarillo bajo temporal, en el norte del país fue de $5.6 \mathrm{t} \mathrm{ha}^{-1}$, mientras que en el sur fue de $2.1 \mathrm{t}$ ha $^{-1}$ (SIAP, 2020). Los datos anteriores implican tener en consideración la tipología de producción que se presenta en estas contrastantes zonas productoras de maíz. Adicionalmente, se puede mencionar que el tipo de semillas que se usan durante la siembra es uno de los ejes medulares de dicho rendimiento (Olguín-López at al., 2017).

En este contexto, se debe tener en cuenta que la producción de este cultivo es un asunto de amplia complejidad, además del impacto que tiene sobre las políticas públicas y soberanía alimentaria del país (Echánove-Huacuja, 2009; Valencia-Romero et al., 2019).

Ante los cambios actuales de las condiciones climáticas, es importante hacer uso de la amplia diversidad que tiene el cultivo, en aras de aprovechar genotipos que sean tolerantes al déficit hídrico y permitan seguir teniendo rendimientos favorables o incluso mejores como los obtenidos hasta ahora con el uso de variedades mejoradas (Espinosa et al., 2009; Virgen et al., 2016; MartínezGutiérrez et al., 2018). El uso generalizado de materiales mejorados ha sido una alternativa viable para incrementar la rentabilidad del cultivo, por lo que es necesaria la formación de nuevos genotipos superiores a los actuales (Ávila-Perches et al., 2009).

Para incrementar los rendimientos de este cultivo, un sistema de mejoramiento ampliamente usado es la hibridación, con ello los híbridos han contribuido al aumento significativo de la producción. La estructura genética que impera en éstos es el uso de tres líneas para formar los llamados híbridos trilineales, también se han usado híbridos dobles y de cruza simple (Márquez-Sánchez, 2009).

Sin embargo, existen cruzamientos no convencionales que por su composición permiten la expresión favorable del vigor híbrido. Por su parte, un diseño genético es un sistema de apareamiento entre individuos de una o más poblaciones que provee información del tipo de acción génica que controla el carácter en cuestión y genera una población mejorada que puede usarse como base para la selección y desarrollo de variedades potenciales (Acquaah, 2012).

La estimación de los parámetros genéticos de una variedad puede hacerse mediante el uso de alguno de los métodos de Griffing (1956), los cuales son de utilidad para los fitomejoradores al emplear progenitores endogámicos o variedades de polinización libre que muestran amplia diversidad genética. En lo particular, el método I analiza a los progenitores usando las cruzas directas y las cruzas recíprocas. 
Permite estimar la aptitud combinatoria general (ACG), la aptitud combinatoria específica (ACE), así como los efectos maternos y recíprocos, que aportan elementos para establecer las relaciones genéticas entre los progenitores y su progenie, siendo útiles en la identificación de híbridos y cruzamientos promisorios (González et al., 2007).

Sprague y Tatum (1942) definieron los términos de aptitud combinatoria general (ACG) y específica (ACE). La primera representa el comportamiento promedio de una línea en función de sus combinaciones híbridas (cruzas), mientras que el segundo término es usado para designar a aquellos casos en los cuales ciertas cruzas son relativamente mejores o peores, sobre la base del comportamiento promedio de los progenitores involucrados. Griffing (1956) estableció que el concepto de aptitud combinatoria es importante en el desarrollo de experimentos en los cuales se estudian y comparan los comportamientos de líneas en combinaciones híbridas, con relación al tipo de acción génica que determina la aptitud combinatoria de las líneas.

Se considera que la ACG se asocia a efectos de aditividad (efectos promedios de los genes), mientras que la ACE a los efectos de dominancia y epistasis (desviaciones promedio) (Ceballos, 1995; Poehlman y Allen, 2003; Hallauer et al., 2010). El objetivo de esta investigación fue determinar el tipo de acción génica involucrada y la influencia de los efectos maternos y recíprocos en el rendimiento y características agronómicas de cruzas dialélicas, directas y recíprocas, de cinco variedades de maíz amarillo y sus respectivos cruzamientos evaluados en tres localidades de los Valles Altos del centro de México.

\section{Materiales y métodos}

\section{Ubicación geográfica}

Los experimentos se establecieron en el ciclo primavera-verano de 2017 a 2019 en tres localidades. La primera, ubicada en el municipio de Cuautitlán Izcalli, Estado de México, la segunda en Santa Lucía de Prías, Coatlinchán, Municipio de Texcoco, Estado de México, donde se encuentra el Campo Experimental del Valle de México del Instituto Nacional de Investigaciones Forestales, Agrícolas y Pecuarias (CEVAMEX-INIFAP) y la tercera en la localidad de Huexotla, Texcoco, Estado de México (Cuadro 1).

Cuadro 1. Evaluación de cinco variedades de maíz amarillo y sus cruzas dialélicas. Primavera verano de 2017 a 2019.

\begin{tabular}{cccc}
\hline Localidades & Ubicación geográfica & Altitud (m) & Suelo (textura) \\
\hline FESC-UNAM & $19^{\circ} 41^{\prime} \mathrm{LN}, 99^{\circ} 11^{\prime} \mathrm{LO}$ & 2274 & Franco arcillosa \\
Santa Lucía de Prías & $19^{\circ} 27^{\prime} \mathrm{LN}, 98^{\circ} 51^{\prime} \mathrm{LO}$ & 2326 & Franco arenosa \\
Huexotla & $19^{\circ} 29^{\prime} \mathrm{LN}, 98^{\circ} 52^{\prime} \mathrm{LO}$ & 2300 & Franco limosa \\
\hline
\end{tabular}

En la Figura 1, se puede apreciar el comportamiento de la precipitación, mientras que en la Figura 2 , se puede ver el comportamiento de las temperaturas medias en las tres localidades de evaluación en el periodo comprendido de mayo a diciembre de 2017 a 2019. 


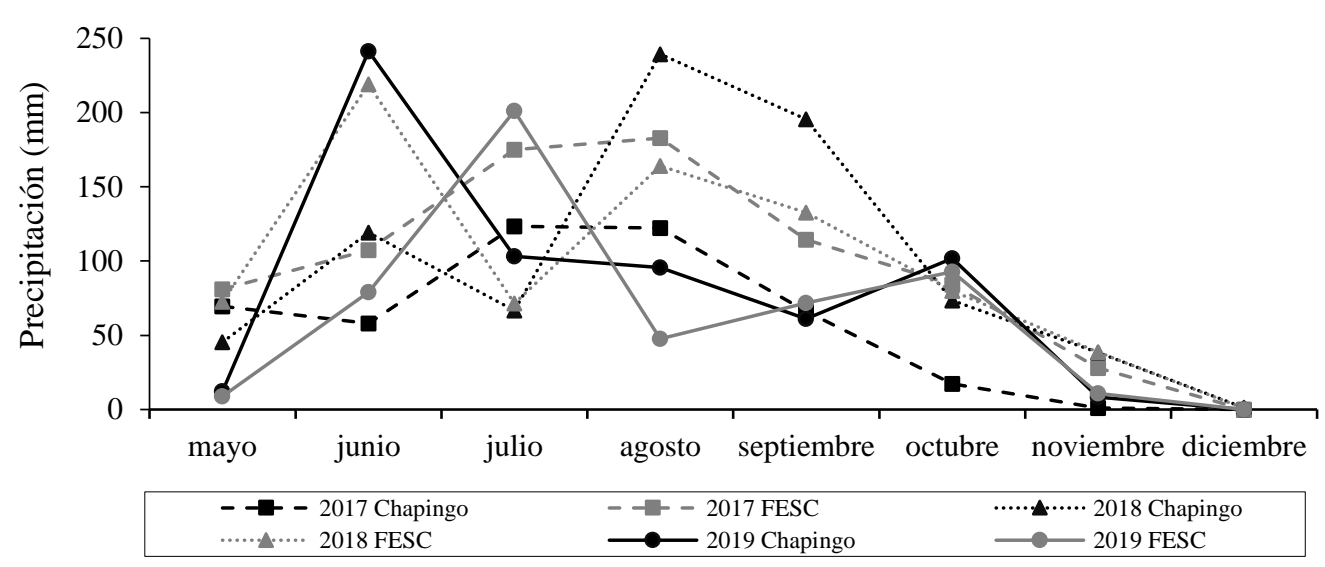

Figura 1. Precipitación (mm) de los ambientes de evaluación. Elaboración con datos de la estación meteorológica de la Universidad Autónoma Chapingo, así como la estación meteorológica Almaraz ubicada en la Facultad de Estudios Superiores Cuautitlán, Campo 4.

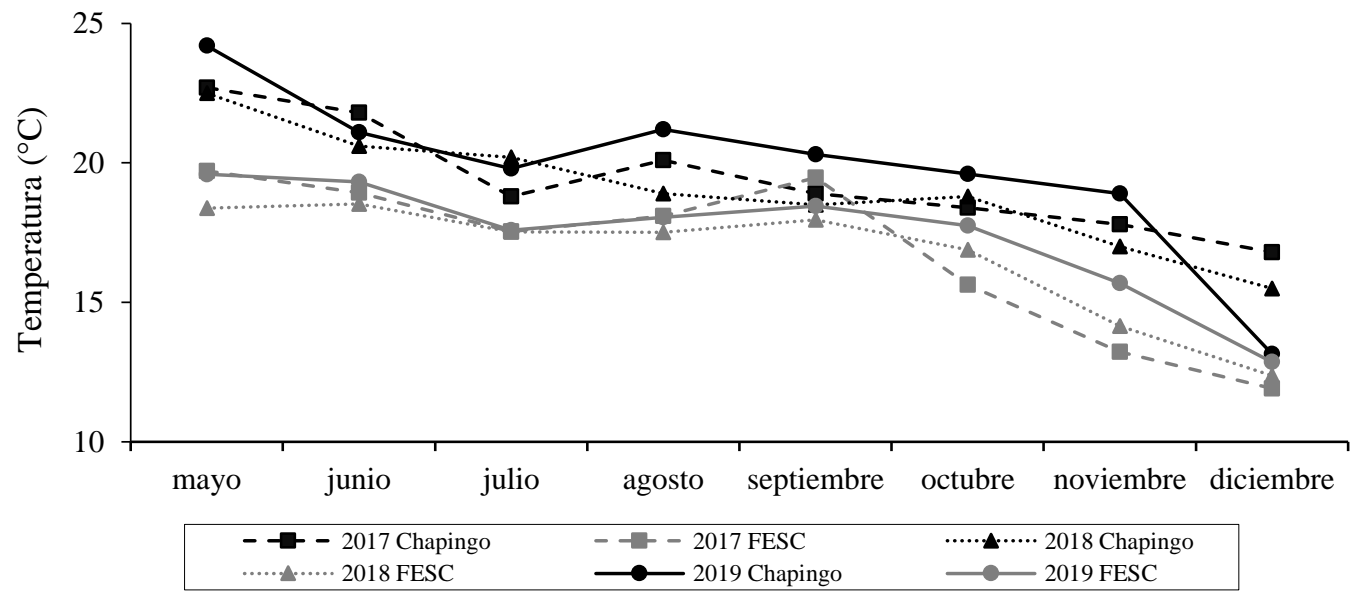

Figura 2. Temperatura media $\left({ }^{\circ} \mathrm{C}\right)$ de los ambientes de evaluación. Elaboración con datos de la estación meteorológica de la Universidad Autónoma Chapingo, así como la estación meteorológica Almaraz de la Facultad de Estudios Superiores Cuautitlán, Campo 4.

En las evaluaciones realizadas en la FESC-UNAM, en el mes de junio el terreno al momento de la siembra presentó humedad residual en los años de evaluación. Únicamente se aplicó un riego de germinación después de la siembra, y el requerimiento hídrico posterior del cultivo se cubrió con la precipitación pluvial ocurrido durante el ciclo del cultivo. Para el caso de Santa Lucía, en el año 2017 se dio un riego después de la siembra y dos riegos de auxilio previo a la floración.

En 2018, en Huexotla había humedad en el suelo al momento de la siembra y se aplicaron tres riegos de auxilio, uno antes de la floración y dos posteriores a ésta. Para 2019, en Santa Lucía también el suelo tenía humedad al momento de la siembra, y se aplicó un riego durante la floración y dos riegos más después de esta etapa; en este año hubo presencia de granizo posterior a la madurez fisiológica del cultivo. Cabe aclarar, que la inconsistencia en el riego en los ambientes de Texcoco fue debido a la poca disponibilidad de agua y fallas en las bombas del Campo Experimental. En los ambientes, la cosecha fue manual y se llevó acabo en el mes de diciembre en los respectivos años de evaluación. 


\section{Material genético}

Durante el ciclo primavera verano de 2016, se realizaron las cruzas dialélicas, directas y recíprocas de las cinco variedades de maíz de grano amarillo (Cuadro 2), cuatro de estas son de germoplasma de Valles Altos (HVAA-10, HVAA-9, E5-6F2 e IA351), obtenidas con la aplicación de tres ciclos de selección masal estratificada.

Cuadro 2. Genealogía de cruzas varietales.

\begin{tabular}{|c|c|c|c|}
\hline Variedad & Progenitor hembra & Progenitor macho & Cruza varietal \\
\hline \multirow[t]{5}{*}{1} & HVAA-10 & HVAA-10 & $1 \times 1$ \\
\hline & & HVAA-9 & $1 \times 7$ \\
\hline & & E5-6F2 & $1 \times 17$ \\
\hline & & IA324 & $1 \times 18$ \\
\hline & & IA351 & $1 \times 19$ \\
\hline \multirow[t]{5}{*}{7} & HVAA-9 & HVAA-10 & $7 \times 1$ \\
\hline & & HVAA-9 & $7 \times 7$ \\
\hline & & E5-6F2 & $7 \times 17$ \\
\hline & & IA 324 & $7 \times 18$ \\
\hline & & IA351 & $7 \times 19$ \\
\hline \multirow[t]{5}{*}{17} & E5-6F2 & HVAA-10 & $17 \times 1$ \\
\hline & & HVAA-9 & $17 \times 7$ \\
\hline & & E5-6F2 & $17 \times 17$ \\
\hline & & IA324 & $17 \times 18$ \\
\hline & & IA351 & $17 \times 19$ \\
\hline \multirow[t]{5}{*}{18} & IA324 & HVAA-10 & $18 \times 1$ \\
\hline & & HVAA-9 & $18 \times 7$ \\
\hline & & E5-6F2 & $18 \times 17$ \\
\hline & & IA324 & $18 \times 18$ \\
\hline & & IA351 & $18 \times 19$ \\
\hline \multirow[t]{5}{*}{19} & IA351 & HVAA-10 & $19 \times 1$ \\
\hline & & HVAA-9 & $19 \times 7$ \\
\hline & & E5-6F2 & $19 \times 17$ \\
\hline & & IA324 & $19 \times 18$ \\
\hline & & IA351 & $19 \times 19$ \\
\hline
\end{tabular}

La variedad IA324 procede de germoplasma de Valles Altos-Transición al que se le aplicaron tres ciclos de selección masal estratificada. Con estos genotipos se generaron 20 cruzas $\mathrm{F}_{1}$; además, se obtuvo semilla de los progenitores por medio de cruzas fraternales. Los 25 genotipos se evaluaron en seis experimentos sembrados en el mes de junio en los años 2017 a 2019. 
Cada fecha de siembra se consideró como un ambiente diferente de evaluación. En los seis ambientes, los genotipos se distribuyeron en un diseño experimental de bloques completos al azar con tres repeticiones. Se utilizó una densidad de población de 65000 plantas ha ${ }^{-1}$, donde la parcela experimental consistió en un surco de $5 \mathrm{~m}$ de largo por $0.8 \mathrm{~m}$ de ancho.

Durante el ciclo de crecimiento y a la fecha de cosecha, se obtuvo información de 20 caracteres cuantitativos asociados con características morfológicas y de componentes de rendimiento, estimados con el valor promedio de cinco mazorcas representativas de cada unidad experimental. Con los datos obtenidos, se realizó un análisis estadístico para identificar las variables de mayor correlación positiva con el rendimiento de grano y realizar la discriminación de variables, quedando definido que los componentes que más correlacionaron con el rendimiento fueron floración masculina (FM; días), altura de planta (AP; cm), longitud de mazorca (Lmz).

Con estas variables, se realizó el análisis genético, el cual se efectuó con el modelo I (de efectos fijos), método I (dialélico completo) de Griffing (1956), el cual examina las líneas parentales, las cruzas $\mathrm{F}_{1}$ directas y recíprocas, para lo cual se utilizó el programa DIALLEL-SAS propuesto por Zhang y Kang (2003), que permite la división de los efectos recíprocos (ER), maternos y no maternos.

La importancia relativa de ACG y ACE fue evaluada con la fórmula [2 x $\left.\mathrm{CM}_{\mathrm{ACG}}\right] /\left[2 \times \mathrm{CM}_{\mathrm{ACG}}+\right.$ $\mathrm{CM}_{\mathrm{ACE}}$ ] (Baker, 1978), donde $\mathrm{CM}_{\mathrm{ACG}}$ es el cuadrado medio de la ACG y $\mathrm{CM}_{\mathrm{ACE}}$ es el cuadrado medio de la ACE.

\section{Resultados y discusión}

\section{Análisis de varianza}

En los cuadrados medios del análisis de varianza combinado (Cuadro 3), se detectaron diferencias altamente significativas $(p \leq 0.01)$ entre ambientes, cruzas, aptitud combinatoria general (ACG), aptitud combinatoria específica (ACE), así como en la interacción ambiente por cruzas. Las diferencias estadísticas entre cruzas se atribuyen a la expresión diferentes de la variación genética entre éstas, asociada con los tipos de acción génica que en cada una se expresó, tales como aditividad y dominancia, así como el resultado de la aditividad e interacción de las variedades progenitoras.

Las diferencias en la expresión entre ambientes indican una situación ambiental cambiante entre años y localidades e indica adaptabilidad de las cruzas ante las diferencias entre las condiciones climáticas, edáficas y de cultivo. Estos contrastes se manifestaron en la interacción significativa ambiente por cruzas, la cual Hallauer et al. (2010) la atribuyen a la estrecha variación de las cruzas simples en comparación con aquellas de más de dos progenitores o de progenitores varietales.

Dadas las diferencias significativas entre las cruzas, la suma de cuadrados fue particionada en la ACG y los efectos maternos de los progenitores, y en la ACE y efectos recíprocos de los cruzamientos. La ACG y la ACE mostraron diferencias altamente significativas $(p \leq 0.01)$ para las variables evaluadas, indicando contrastes genéticos debidos a efectos aditivos y no aditivos, respectivamente. 
Baker (1978) sugirió una relación entre ACG y ACE (ACG:ACE), para inferir la importancia de estas sobre el comportamiento de la progenie. Valores cercanos a la unidad, indican una mayor probabilidad del comportamiento basado solamente en la ACG.

Para esta investigación, la contribución de los cuadrados medios de la ACG a la variación fue superior a la presentada por la ACE para todas las variables, con excepción de altura de planta y longitud de mazorca. Para el resto de las variables, la contribución de la ACG fue de $80 \%$ (rendimiento), 79\% (floración masculina) y 91\% (peso volumétrico) (Cuadro 3).

Cuadro 3. Cuadrados medios del análisis de varianza combinado para rendimiento y otras variables agronómicas en un sistema de cruzas dialélicas completo.

\begin{tabular}{ccccccc}
\hline Factor de variación & GL & Rendimiento & FM & AP & PV & LMz \\
\hline Ambiente & 5 & $599365446^{* *}$ & $361.9^{* *}$ & $40962.4^{* *}$ & $315^{* *}$ & $79^{* *}$ \\
Cruzas & 24 & $17561889^{* *}$ & $22.2^{* *}$ & $572.7^{* *}$ & $62^{* *}$ & $6.2^{* *}$ \\
ACG & 4 & $38599842^{* *}$ & $30.2^{* *}$ & $648^{* *}$ & $212^{* *}$ & $5^{* *}$ \\
ACE & 10 & $19789076^{* *}$ & $15.6^{* *}$ & $696^{* *}$ & $44^{* *}$ & $9^{* *}$ \\
EMat & 4 & $13881918^{* *}$ & $58.7^{* *}$ & $607^{* *}$ & $30^{* *}$ & $6.9^{* *}$ \\
ERec & 10 & $6919520^{* *}$ & $25.7^{* *}$ & $419^{* *}$ & $19.4^{* *}$ & $4^{* *}$ \\
Amb x cruzas & 120 & $3717342^{* *}$ & $4^{* *}$ & $337.9^{* *}$ & $15.5^{* *}$ & $2.1^{* *}$ \\
Amb x ACG & 4 & $3603456^{* *}$ & $5.8^{*}$ & $1813^{* *}$ & $34.7^{* *}$ & 0.97 \\
Amb x ACE & 10 & $3516460^{* *}$ & $5.5^{* *}$ & $535^{* *}$ & $19.8^{* *}$ & 0.32 \\
Error & 288 & 269472 & 1.3 & 78.2 & 1.58 & 1.04 \\
CV (\%) & & 8.3 & 1.5 & 4.09 & 1.67 & 6.9 \\
Media & & 6219 & 76 & 215.7 & 75.5 & 15 \\
ACG:ACE & & 0.8 & 0.79 & 0.65 & 0.91 & 0.53 \\
\hline
\end{tabular}

** , * $=p \leq 0.01$ y $p \leq 0.05$, respectivamente; $\mathrm{GL}=$ grados de libertad; $\mathrm{ACG}=$ aptitud combinatoria general; $\mathrm{ACE}=$ aptitud combinatoria específica; EMat $=$ efectos maternos; $E R e c=$ efectos recíprocos; $A m b=$ ambiente; $F M=$ floración masculina; $\mathrm{AP}=$ altura de planta; $\mathrm{PV}=$ peso volumétrico; $\mathrm{LMz}=$ longitud de mazorca; $\mathrm{CV}=$ coeficiente de variación.

La proporción relativa de los efectos de ACG y ACE determinada por los cuadrados medios, indica el tipo de acción génica (Antuna et al., 2003), donde la ACG está asociada principalmente con efectos aditivos y la ACE a efectos no aditivos, por lo tanto, con los resultados se puede inferir que la varianza genética aditiva fue mayor que la no aditiva. En general, el análisis de los cuadrados medios muestra que los efectos genéticos aditivos (ACG) fueron de mayor expresión para rendimiento del grano, floración masculina, peso volumétrico y número de hileras por mazorca.

Los efectos maternos (EMat) fueron altamente significativos $(p \leq 0.01)$; es decir, las características evaluadas (rendimiento, floración masculina, altura de planta, peso volumétrico y longitud de mazorca) estuvieron determinadas estadísticamente por efectos nucleares y citoplasmáticos, lo que significa que las cruzas pueden realizarse y utilizarse en ambos sentidos (directo y recíproco). Los efectos recíprocos (ERec) mostraron diferencias altamente significativas $(p \leq 0.01)$, lo que se atribuye a los afectos de interacción entre el DNA nuclear y el citoplásmico (Sánchez-Hernández et al., 2011), los cuales fueron denominados por Cockerham (1963) como efectos no maternos. 
La interacción significativa Amb x cruzas, condicionó la división de los efectos de interacción de Amb x ACG y Amb x ACE, las cuales también fueron estadísticamente significativas $(p \leq 0.01)$, excepto para floración masculina y longitud de mazorca.

\section{Expresión fenotípica y efecto de ACG de las variedades}

Se encontraron diferencias altamente significativas $(p \leq 0.01)$ para cada variable (Cuadro 4$)$, pero no así en todos los progenitores evaluados. Para rendimiento la variedad cuatro (IA324; $7163 \mathrm{~kg}$ $\mathrm{ha}^{-1}$ ) fue la que expresó el valor más alto para esta variable, seguido por las variedades HVAA-10(6 $\left.533.9 \mathrm{~kg} \mathrm{ha}^{-1}\right)$, E5-6F2- (5 $\left.673 \mathrm{~kg} \mathrm{ha}^{-1}\right)$, IA351 (5 $\left.735 \mathrm{~kg} \mathrm{ha}^{-1}\right)$ y HVAA-9- (3 $\left.761 \mathrm{~kg} \mathrm{ha}^{-1}\right)$.

Estos resultados indican que la variedad cuatro (IA324), tendrá una alta contribución en la expresión del rendimiento de su progenie y que los efectos aditivos son los más importantes, por lo tanto, podría incluirse en un programa de mejoramiento genético de maíz por selección, para contribuir con alelos favorables para esta variable (Guillén-de la Cruz et al., 2009).

Todos los progenitores presentaron valores deseables para las diversas variables evaluadas, con especial énfasis en floración masculina (-0.614) para IA351 (74 días a FM), altura de planta (1.74) para IA324 $(216 \mathrm{~cm})$, peso volumétrico para el progenitor HVAA-9- $\left(70 \mathrm{~kg} \mathrm{hl}^{-1}\right)$, longitud de mazorca (-2.26) para E5-6F2- (15 cm) (Cuadro 4).

Cuadro 4 Efecto de la aptitud combinatoria general (ACG) para rendimiento de grano y otras variables agronómicas de cinco variedades de grano amarillo evaluadas en seis ambientes. Primavera-verano 2017-2019.

\begin{tabular}{cccccc}
\hline Variedad & Rendimiento & FM & AP & PV & LMz \\
\hline HVAA-10 & -42.5 & -0.131 & $1.66^{*}$ & $-0.75^{* *}$ & 0.056 \\
HVAA-9 & $-383.6^{* *}$ & $0.474^{* *}$ & -0.99 & $-1.39^{* *}$ & 0.1 \\
E5-6F2 & $-119.2^{*}$ & $0.219^{*}$ & 0.31 & 0.063 & $-2.26^{* *}$ \\
IA324 & $795.11^{* *}$ & 0.052 & $1.74^{*}$ & $1.21^{* *}$ & $0.15^{*}$ \\
IA351 & $-249.8^{* *}$ & -0.614 & $-2.73^{* *}$ & $0.86^{* *}$ & -0.049 \\
\hline
\end{tabular}

**,$\stackrel{*}{=} p \leq 0.01 \mathrm{y} p \leq 0.05$, respectivamente; $\mathrm{FM}=$ floración masculina; $\mathrm{AP}=$ altura de planta; $\mathrm{PV}=$ peso volumétrico; $\mathrm{LMz}=$ longitud de mazorca.

Para los efectos maternos de los progenitores se observa que los valores superiores (positivos) para rendimiento y sus componentes indican que los progenitores pueden expresar su potencial en las variables evaluadas en el caso de sus cruzas directas (Cuadro 5); es decir, cuando se usan exclusivamente como progenitor femenino.

En los casos de los progenitores HVAA-10 e IA351 presentaron valores negativos para rendimiento, por lo que se espera que sus cruzas directas se comporten desfavorablemente. Estos progenitores cuando se empleen como hembra, su progenie mostrará detrimento en esta característica, pero con valores altos en las otras variables como lo mencionan Núñez-Terrones et al. (2019). 
Cuadro 5. Efectos maternos de cinco variedades de maíz de grano amarillo evaluadas en seis ambientes. Primavera-verano 2017 a 2019.

\begin{tabular}{lccccc}
\hline \multicolumn{1}{c}{ Variedad } & Rendimiento & FM & AP & PV & LMz \\
\hline HVAA-10 & $-222.2^{* *}$ & $0.23^{*}$ & $2.89^{* *}$ & -0.04 & $0.32^{* *}$ \\
HVAA-9 & $-150^{*}$ & $0.59^{* *}$ & -3.4 & 0.19 & -0.13 \\
E5-6F2 & $601.6^{* *}$ & $0.22^{*}$ & -0.2 & $-0.68^{* *}$ & $0.19^{*}$ \\
IA324 & -10 & $0.18^{*}$ & 1 & -0.17 & $-0.26^{* *}$ \\
IA351 & $-219.4^{* *}$ & $-1.24^{* *}$ & -0.28 & $0.7^{* *}$ & -0.13 \\
\hline
\end{tabular}

${ }^{* *},{ }^{*}=p \leq 0.01$ y $p \leq 0.05$, respectivamente; $\mathrm{FM}=$ floración masculina; $\mathrm{AP}=$ altura de planta; $\mathrm{PV}=$ peso volumétrico; $\mathrm{LMz}=$ longitud de mazorca; $\mathrm{HMz}=$ hileras por mazorca.

\section{Expresión fenotípica y efecto de la ACE de los híbridos varietales}

El efecto de la ACE para los componentes de rendimiento fue variable para la mayoría de las cruzas (Cuadro 6), nueve de las cruzas directas fueron altamente significativas $(p \leq 0.01)$, su rendimiento fue de 4759 a $7490 \mathrm{~kg} \mathrm{ha}^{-1}$. Las cruzas directas con mayor ACE para la variable rendimiento fueron 1 x $18\left(7490 \mathrm{~kg} \mathrm{ha}^{-1}\right)$ y 7 x 18 (7 $\left.272 \mathrm{~kg} \mathrm{ha}^{-1}\right)$ (HVAA-10- x IA324 y HVAA-9- x IA324).

Cuadro 6. Efecto de aptitud combinatoria específica (ACE) de 10 cruzas directas y 10 recíprocas, en el cruzamiento de cinco variedades de maíz amarillo, para rendimiento de grano y componentes, evaluadas en seis ambientes. Primavera-verano 2017 a 2019.

\begin{tabular}{|c|c|c|c|c|c|c|c|}
\hline \multirow{2}{*}{ Tipo de cruza } & \multirow{2}{*}{ HVE } & \multicolumn{2}{|c|}{ Rendimiento } & \multicolumn{2}{|c|}{ Floración masculina } & \multicolumn{2}{|c|}{ Altura de planta } \\
\hline & & $\mathrm{ACE}$ & $\mathrm{kg} \mathrm{ha}^{-1}$ & $\mathrm{ACE}$ & días & $\mathrm{ACE}$ & $\mathrm{cm}$ \\
\hline $\mathrm{CD}$ & $1 \times 7$ & $-774.9^{* *}$ & 4759 & -0.47 & 76 & -1.73 & 216 \\
\hline $\mathrm{CR}$ & $7 \times 1$ & $-259.6^{*}$ & 5278 & 0.19 & 76 & 1.61 & 213 \\
\hline $\mathrm{CD}$ & $1 \times 17$ & $-288.5^{* *}$ & 5061 & -0.047 & 76 & -0.68 & 221 \\
\hline $\mathrm{CR}$ & $17 \times 1$ & $-708.1^{* *}$ & 6477 & -0.3 & 77 & $4.25^{*}$ & 213 \\
\hline $\mathrm{CD}$ & $1 \times 18$ & $378.82^{* *}$ & 7490 & $0.34^{*}$ & 76 & -0.73 & 224 \\
\hline $\mathrm{CR}$ & $18 \times 1$ & 138.8 & 7212 & -0.028 & 77 & $5.3^{* *}$ & 213 \\
\hline $\mathrm{CD}$ & $7 \times 17$ & $725.08^{* *}$ & 6112 & -1.09 & 76 & $3.5^{*}$ & 215 \\
\hline $\mathrm{CR}$ & $17 \times 7$ & $-330.1^{*}$ & 6772 & $0.639^{*}$ & 75 & $-3.38^{*}$ & 222 \\
\hline $\mathrm{CD}$ & $7 \times 18$ & $1075.29^{* *}$ & 7272 & -0.09 & 77 & $5.26^{* *}$ & 216 \\
\hline $\mathrm{CR}$ & $18 \times 7$ & $-434.2^{* *}$ & 8140 & 0.36 & 76 & $-6.08^{* *}$ & 228 \\
\hline $\mathrm{CD}$ & $17 \times 18$ & 150 & 7430 & -0.28 & 76 & -1.98 & 216 \\
\hline $\mathrm{CR}$ & $18 \times 17$ & $519.88^{* * *}$ & 6390 & -0.08 & 76 & 0.3 & 215 \\
\hline $\mathrm{CD}$ & $1 \times 19$ & $285.22^{* *}$ & 6152 & 0.29 & 77 & 3.2 & 218 \\
\hline $\mathrm{CR}$ & $19 \times 1$ & -60 & 6272 & $1.028^{* *}$ & 75 & 0.41 & 217 \\
\hline $\mathrm{CD}$ & $7 \times 19$ & $665.44^{* *}$ & 6156 & -0.013 & 78 & $5.3^{* *}$ & 215 \\
\hline $\mathrm{CR}$ & $19 \times 7$ & -95.2 & 6347 & $1.61^{* *}$ & 74 & -2.52 & 220 \\
\hline $\mathrm{CD}$ & $17 \times 19$ & $-143.5^{*}$ & 6555 & 0.19 & 77 & $-2.69^{*}$ & 210 \\
\hline $\mathrm{CR}$ & $19 \times 17$ & $848.3^{* *}$ & 4859 & $1.33^{* *}$ & 75 & -0.27 & 211 \\
\hline $\mathrm{CD}$ & $18 \times 19$ & $-822.7^{* *}$ & 6126 & 0.29 & 77 & 0.17 & 218 \\
\hline $\mathrm{CR}$ & $19 \times 18$ & $184.5^{*}$ & 5758 & $1^{* *}$ & 75 & $3.52^{*}$ & 211 \\
\hline
\end{tabular}

$*^{* *},{ }^{*}=p \leq 0.01$ y $p \leq 0.05$, respectivamente; $\mathrm{HVE}=$ híbrido varietal experimental; $\mathrm{CD}=$ cruza directa; $\mathrm{CR}=$ cruza recíproca; $\mathrm{FM}=$ floración masculina; $\mathrm{AP}=$ altura de planta; $\mathrm{PV}=$ peso volumétrico; $\mathrm{LMz}=$ longitud de mazorca; $\mathrm{ACE}=$ aptitud combinatoria específica. 
En el primer caso, la cruza con mayor ACE y rendimiento fue el resultado de cruzar dos progenitores de alta $\mathrm{ACG}$, la segunda cruza sobresaliente fue el resultado de cruzar un progenitor de baja con uno de alta ACG, lo que responde a lo señalado por Escorcia et al. (2010) y Guerrero et al. (2011), quienes señalan que una cruza simple es de alto rendimiento cuando sus progenitores son de alta ACG o bien, al menos uno de ellos lo es, pero presentan efectos altos positivos de ACE.

La cruza 7 x 18 también resultó sobresaliente por su ACE en las variables de altura de planta (Cuadro 6) y peso volumétrico Cuadro 7, estas variables presentaron diferencias altamente significativas $(p \leq 0.01)$. Por su parte, cuatro cruzas recíprocas presentaron diferencias altamente significativas y tres fueron significativas $(p \leq 0.01 \mathrm{y} p \leq 0.05)$, con rendimientos de 4859 a 8140 $\mathrm{kg} \mathrm{ha}^{-1}$. La cruza recíproca con mayor ACE para la variable rendimiento fue $18 \mathrm{x} 7$ (IA324 x HVAA-9). La cruza directa 17 x 18 (E5-6F2 x IA324) y la cruza recíproca 18 x 1 (IA324 x HVAA10) aunque no presentaron diferencias altamente significativas, su rendimiento fue de los más altos (7 490, 7430 y $7212 \mathrm{~kg} \mathrm{ha}^{-1}$, respectivamente), estas cruzas son el resultado del cruzamiento entre dos progenitores de alta ACG.

Cuadro 7. Efecto de aptitud combinatoria específica (ACE) de 10 cruzas directas y 10 cruzas recíprocas, en cruzamiento de cinco variedades de maíz amarillo, para componentes de rendimiento, evaluadas en seis ambientes. Primavera verano 2017 a 2019.

\begin{tabular}{|c|c|c|c|c|c|}
\hline \multirow{2}{*}{ Tipo de cruza } & \multirow{2}{*}{ HVE } & \multicolumn{2}{|c|}{ Peso volumétrico } & \multicolumn{2}{|c|}{ Longitud de mazorca } \\
\hline & & $\mathrm{ACE}$ & $\left(\mathrm{kg} \mathrm{hl}^{-1}\right)$ & $\mathrm{ACE}$ & $\mathrm{cm}$ \\
\hline $\mathrm{CD}$ & $1 \times 7$ & -0.24 & 73 & 0.22 & 15 \\
\hline $\mathrm{CR}$ & $7 \times 1$ & -0.19 & 73 & 0.14 & 15 \\
\hline $\mathrm{CD}$ & $1 \times 17$ & $-0.83^{* *}$ & 75 & $-0.71^{* *}$ & 14 \\
\hline $\mathrm{CR}$ & $17 \times 1$ & $1.28^{* *}$ & 73 & 0.055 & 14 \\
\hline $\mathrm{CD}$ & $1 \times 18$ & -0.12 & 75 & -0.11 & 15 \\
\hline $\mathrm{CR}$ & $18 \times 1$ & $-0.8^{* *}$ & 77 & $0.47^{* *}$ & 15 \\
\hline $\mathrm{CD}$ & $7 \times 17$ & $0.77^{\text {** }}$ & 75 & $0.32^{*}$ & 15 \\
\hline $\mathrm{CR}$ & $17 \times 7$ & $0.47^{*}$ & 74 & -0.19 & 15 \\
\hline $\mathrm{CD}$ & $7 \times 18$ & $1.4^{* *}$ & 77 & $0.43^{*}$ & 16 \\
\hline $\mathrm{CR}$ & $18 \times 7$ & 0.25 & 76 & 0.22 & 15 \\
\hline $\mathrm{CD}$ & $17 \times 18$ & $0.64^{* *}$ & 77 & 0.15 & 15 \\
\hline $\mathrm{CR}$ & $18 \times 17$ & -0.11 & 78 & 0.25 & 15 \\
\hline $\mathrm{CD}$ & $1 \times 19$ & 0.13 & 75 & $0.31^{*}$ & 16 \\
\hline $\mathrm{CR}$ & $19 \times 1$ & $-0.44^{*}$ & 76 & $0.64^{* *}$ & 15 \\
\hline $\mathrm{CD}$ & $7 \times 19$ & $0.98^{* *}$ & 76 & $0.44^{* *}$ & 15 \\
\hline $\mathrm{CR}$ & $19 \times 7$ & -0.14 & 76 & $-0.42^{*}$ & 16 \\
\hline $\mathrm{CD}$ & $17 \times 19$ & 0.16 & 76 & -0.14 & 15 \\
\hline $\mathrm{CR}$ & $19 \times 17$ & $-0.88^{* *}$ & 77 & $0.36^{*}$ & 14 \\
\hline $\mathrm{CD}$ & $18 \times 19$ & $-1.37^{* * *}$ & 75 & -0.12 & 15 \\
\hline $\mathrm{CR}$ & $19 \times 18$ & $-1.22^{* *}$ & 78 & -0.08 & 15 \\
\hline
\end{tabular}

$*^{* *},{ }^{*}=p \leq 0.01$ y $p \leq 0.05$, respectivamente; $\mathrm{CD}=$ cruza directa; $\mathrm{CR}=$ cruza recíproca; $\mathrm{PV}=$ peso volumétrico; $\mathrm{LMz}=$ longitud de mazorca; $\mathrm{HMz}=$ hileras por mazorca; $\mathrm{ACE}=$ aptitud combinatoria específica. 
Para días a floración masculina para las cruzas que presentaron diferencias altamente significativas $(p \leq 0.01)$ el efecto aditivo fue el que imperó en sus progenies cuando el progenitor IA351 se usó como hembra; la que per se fue la variedad de grano amarillo más precoz dentro de los progenitores evaluados (74 días a floración masculina) y esto se vio reflejado en sus cruzas directas (74 a 75 días a FM), cuando esta variedad se utiliza como macho; es decir, si se realizan cruzas recíprocas, se atrasa la floración por tres a cuatro días (77 a 78 días a FM).

Para la variable peso volumétrico, la presencia del progenitor V2 (HVAA-9-) reduce la densidad del grano de su progenie (73 a $75 \mathrm{~kg} \mathrm{hl}^{-1}$, valores inferiores al promedio general), a pesar que la ACE de dos de sus cruzas directas sea altamente significativo (0.77 y 0.98), con excepción de la cruza directa 7 x 18, donde se mantiene la densidad del progenitor IA324 (77 kg hl-1) que funge como progenitor macho, pero ocurre lo contrario; es decir, la densidad disminuye cuando IA324 se utiliza como hembra $\left(76 \mathrm{~kg} \mathrm{hl}^{-1}\right)$, aunque se mantiene el valor promedio general (Cuadro 7).

Los efectos recíprocos (ERec) son un factor relevante en el mejoramiento genético del maíz, en donde la expresión de estos efectos dados por la diversidad genética de los progenitores se debe tener en cuenta (Khehra y Bhalla, 1976).

\section{Conclusiones}

En los híbridos varietales, los efectos de aptitud combinatoria general (efectos aditivos) fueron más importantes que los de aptitud combinatoria específica en la mayoría de los caracteres agronómicos. Hubo presencia de efectos maternos y recíprocos; por lo tanto, las características evaluadas estuvieron determinadas por herencia nuclear y citoplásmica, lo que permitió el desarrollo y aprovechamiento de la cruza directa y recíproca 7 x 18 y 18 x 7, así como 1 x 18 y 18 $x$ 1. La cruza directa con mayor ACE para la variable rendimiento fue HVAA-10 x IA324. La segunda cruza directa HVAA-9 x IA324 fue sobresaliente por su ACE para rendimiento, altura de planta y peso volumétrico. La cruza recíproca con mayor ACE para rendimiento fue 18 x 7 (IA324 x HVAA-9).

El empleo de progenitores de ACG contrastante (alta y baja) permitió la expresión de sus progenies con características agronómicas favorables. Las variedades de grano amarillo con altos efectos de ACG pueden emplearse para desarrollar variedades sintéticas o seguir avanzando más ciclos de mejoramiento, teniendo en cuenta que las líneas con alta ACG observadas en pruebas tempranas conservan sus valores aditivos en generaciones avanzadas, mientras que las cruzas con alta ACE se pueden usar para hibridación.

\section{Agradecimientos}

Se agradece el apoyo financiero de la UNAM a través del Programa de Apoyo a Proyectos de Investigación e Innovación Tecnológica (PAPIIT): IT201618.

\section{Literatura citada}

Acquaah, G. 2012. Principles of plant genetics and breeding. 2a (Ed.). Wiley-Blackwell, Oxford. $740 \mathrm{p}$. 
Antuna, G.; Rincón, F.; Gutiérrez, E.; Ruiz, N. y Bustamante, L. 2003. Componentes genéticos de caracteres agronómicos y de calidad fisiológica de semillas de líneas de maíz. Rev. Fitotec. Mex. 26(1):11-17.

Ávila-Perches, M. Á.; Rodríguez-Herrera, S. A.; Vázquez-Badillo, M. E.; Borrego-Escalante, F.; Lozano-del Río, A. J. y López-Benítez, A. 2009. Aptitud combinatoria y efectos recíprocos en líneas endogámicas de maíz de Valles Altos del centro de México. Agric. Téc. Méx. 35(3):285-293.

Baker, R. 1978. Issues in diallel analysis. Crop Sci. 18(4):533-536. https://doi.org/10.2135/ cropsci1978.0011183X001800040001x.

Ceballos, H. 1995. Principios básicos de genética cuantitativa. Palmira, Universidad Nacional. 254 p.

Cockerham, C. C. 1963. Estimation of genetics variances. In: Hanson, W. D. and Robinson, H. F (eds). Statistical and Plant Breeding. NAS-NRC. 982. Washington, DC. 53-93 pp.

Echánove-Huacuja, F. 2009. Políticas públicas y maíz en México: el esquema de agricultura por contrato. Anales de Geografía. 29(2):65-82.

Escorcia, G. N.; Molina, G. J.; Castillo, G. F. y Mejía, C. J. 2010. Rendimiento, heterosis y depresión endogámica de cruzas simples de maíz. Rev. Fitotec. Mex. 33(3):271-279.

Espinosa, A.; Tadeo, M.; Turrent, A. y Gómez, N. 2009. El potencial de las variedades nativas y mejoradas de maíz. Ciencias. 92(93):118-125.

González, H. A.; Pérez, L. D.; Sahagún, C. J.; Norman, M. T. H.; Balbuena, M. A.; Gutiérrez, R. F. 2007. Análisis de una cruza dialélica completa de líneas endogámicas de maíz. Ciencias Agrícolas Informa. 16(1):10-17.

Griffing, B. 1956. Concept of general and specific combining ability in relation to diallel crossing systems. Austr. J. Biol. Sci. 9(4):463-493.

Guerrero, C.; Espinoza, A.; Palomo, A.; Gutiérrez, E.; Zermeño, H. y González, M. P. 2011. Aptitud combinatoria del rendimiento y sus componentes en dos grupos de líneas de maíz. Agron. Mesoam. 22(2):257-267.

Guillén-de La Cruz, P.; de La Cruz-Lázaro, E.; Castañón-Nájera, G.; Osorio-Osorio, R.; BritoManzano, N. P.; Lozano-del Río, A. y López-Noverola, U. 2009. Aptitud combinatoria general y específica de germoplasma tropical de maíz. Trop. Subtrop. Agroecosys. 10(1):101-107.

Hallauer, A.; Carena, R. M. and Miranda, J. B. 2010. Quantitative genetics in maize breeding. Springer-Verlag. New York Inc. 664 p.

Khehra, A. S. and Bhalla, S. K. 1976. Cytoplasmic effects on quantitative characters in maize (Zea mays L.). Theor. Appl. Genet. 47(6):271-274. https://doi.org/10.1007/BF00281931.

Márquez-Sánchez, F. 2009. De las variedades criollas de maíz a los híbridos transgénicos. II: la hibridación. Agric. Soc. Des. 6(2):161-176.

Martínez-Gutiérrez, A.; Zamudio-González, B.; Tadeo-Robledo, M.; Espinosa-Calderón, A.; Cardoso-Galvão, J. C.; Vázquez-Carrillo, G. y Turrent-Fernández, A. 2018. Rendimiento de híbridos de maíz grano blanco en cinco localidades de Valles Altos de México. Rev. Mex. Cienc. Agríc. 9(7):1447-58.

Núñez-Terrones, E.; Mendoza-Castillo, M. D.; Delgado-Alvarado, A.; Castillo-González, F. y Sánchez-Ramírez, F. J. 2019. Análisis genético de componentes nutricionales en cruzas simples de maíz de grano blanco. Rev. Fitotec. Mex. 42(2):163-172.

Olguín-López, J. L.; Guevara-Gutiérrez, R. D.; Carranza-Montaño, J. A.; Scopel, E.; BarretoGarcía, O. A.; Mancilla-Villa, O. R. y Talavera-Villareal, A. 2017. Producción y rendimiento de maíz en cuatro tipos de labranza bajo condiciones de temporal. IDESIA. 35(1):51-61. 
Poehlman, J. M. y Allen, D. 2003. Mejoramiento genético de las cosechas. Editorial Limusa. México, D. F. 200 p.

Sánchez-Hernández, C.; Villanueva-Verduzco, C.; Sahagún-Castellanos, J.; Martínez-Solís, J.; Legaria-Solano, J. P. y Sánchez-Hernández, M. A. 2011. Efectos de aptitud combinatoria en híbridos de calabacita tipo Grey Zucchini. Rev. Chapingo Ser. Hortic. 17(2):89-103.

SIAP. 2020. Servicio de Información Agroalimentaria y Pesquera. Anuario Estadístico de la Producción Agrícola. https://nube.siap.gob.mx/cierreagricola/.

Sprague, G. F. and Tatum, L. A. 1942. General versus specific combining ability in single crosses of corn. J. Am. Soc. Agron. 34(10):264-275. https://doi.org/10.2134/agronj1942. $00021962003400100008 x$.

Valencia-Romero, R.; Sánchez-Bárcenas, H. y Robles-Ortiz, D. 2019. Soberanía Alimentaria de granos básicos en México: un enfoque de cointegración de Johansen a partir del TLCAN. Análisis Econ. 37(87):223-248.

Virgen, V. J.; Zepeda, B. R.; Ávila, P. M. A.; Espinosa, C. A.; Arellano, V. J. L. y Gámez, V. A. 2016. Producción y calidad de semillas de maíz en Valles Altos de México. Agron. Mesoam. 27(1):191-206.

Zhang, Y. and Kang, M. S. 2003. DIALLEL-SAS: a program for Griffing's diallel methods. In M.S. Kang, (Ed). Handbook of formulas and software for plants geneticists and breeders. Food Products Press, New York, USA. 1-19 pp. 